\title{
30 kV STEM-SEM - The Perfect Conditions for Transmission Spectroscopy?
}

Sam Marks ${ }^{1}$, Philippe Pinard ${ }^{2}$, Sharhid Jabar ${ }^{3}$, Geoff West ${ }^{3}$, George Wetzel ${ }^{4}$, Simon Burgess ${ }^{1}$ and Christian Lang $^{5}$

${ }^{1}$ Oxford Instruments NanoAnalysis, High Wycombe, England, United Kingdom, ${ }^{2}$ Oxford Instruments NanoAnalysis, High Wycombe, United Kingdom, ${ }^{3}$ Warwick Manufacturing Group, University of Warwick, United States, ${ }^{4}$ Clemson University, United States, ${ }^{5}$ Oxford Instruments Nanoanalysis, High Wycombe, England, United Kingdom

Scanning Electron Microscopy (SEM) has seen continuous improvements to spatial resolution at both high, $30 \mathrm{kV}$, and low, $1 \mathrm{kV}$, accelerating voltages over the last 30 years. This has driven the demand for high spatial resolution Energy Dispersive Spectroscopy (EDS) spectral imaging to match the spatial resolution specification of the microscope. Improvements in EDS technology, transitioning from SiLi detectors to Silicon Drift Detectors (SDD), have resulted in greater X-ray detection efficiency. However, spatial resolution in spectral images is predominantly governed by the interaction volume, a measure of electron penetration and X-ray generation, and not the detection efficiency.

Under normal $20 \mathrm{kV}$ SEM high-resolution imaging conditions, due to the large interaction volume, the spatial resolution of spectral images will be orders of magnitude larger than the size of the electron spot (Figure 1a). Reducing the accelerating voltage will reduce the interaction volume and improve the spatial resolution of the spectral images. Sub $10 \mathrm{~nm}$ EDS spatial resolution has been achieved using windowless EDS detection at short working distances and with an accelerating voltage of $1.5 \mathrm{kV}$ [1].

Alternatively, the spatial resolution of spectral images can be improved by utilising electron transparent samples. The lateral broadening of the interaction volume is proportional to the depth of penetration, by reducing sample thickness to $<100 \mathrm{~nm}$ we can greatly improve the spatial resolution at high accelerating voltages. Monte-Carlo simulations of the interaction volume calculated using NISTMonte [2], indicate that $>70 \%$ of the X-rays generated at $30 \mathrm{kV}$ originate from within the first $10 \mathrm{~nm}$ of sample (Figure 1c). Therefore, high-resolution spectral images of electron transparent samples at $30 \mathrm{kV}$ can achieve spatial resolutions similar to both high-resolution $200 \mathrm{kV} \mathrm{STEM}$ and high-resolution $1.5 \mathrm{kV}$ bulk SEM [3].

We have investigated the spectral imaging spatial resolutions achievable for a range of accelerating voltages between 1 and $200 \mathrm{kV}$. Results highlight that STEM-SEM of electron transparent samples can be utilised to acquire the best spatial resolution spectrum images from an SEM. Furthermore, we have modelled the theoretical X-ray emission as a function of solid angle, beam current and sample thickness, identifying that, for a $100 \mathrm{~nm}$ thick sample, $30 \mathrm{kV}$ is a more efficient accelerating voltage for X-ray emission than $200 \mathrm{kV}$. A result of the interaction probability, with high energy electrons more likely to travel through the sample without any inelastic interaction. These calculations indicate that under the same acquisition settings, a $200 \mathrm{kV}$ TEM would require twice the solid angle to achieve equivalent $\mathrm{X}$-ray count rates to a $30 \mathrm{kV}$ STEM-SEM (Figure 2).

A bulk steel sample, known to include sub $100 \mathrm{~nm}$ V-rich inclusions, was measured using a Thermo Scientific Scios FIB-SEM equipped with an Oxford Instruments X-Max ${ }^{N}$ EDS detector. Initial experiments were performed using $20 \mathrm{kV}$ SEM, at this accelerating voltage, the V spatial resolution was found to $\mathrm{be} \approx 100 \mathrm{~nm}$, with no smaller inclusions visible against the background EDS signal (Figure 1b). 
Using a FIB-SEM, a sample was extracted from the bulk and thinned to approximately $100 \mathrm{~nm}$ before being imaged using STEM-SEM at $30 \mathrm{kV}$ (Figure 1d). This resulted in a large spatial resolution improvement, bringing the characterisation capabilities of V-rich inclusions from $100 \mathrm{~nm}$ to $20 \mathrm{~nm}$. The workflow to analyse an electron transparent sample using STEM-SEM was found to require less time and expertise than that of the workflow to analysing an electron transparent sample in a TEM. The ease of use of STEM-SEM combined with the resolution improvements and enhanced X-ray collection efficiency make STEM-SEM an extremely powerful tool for high spatial resolution spectral imaging. We feel proper utilisation of STEM-SEM could reduce the TEM workload for high magnification EDS analysis.
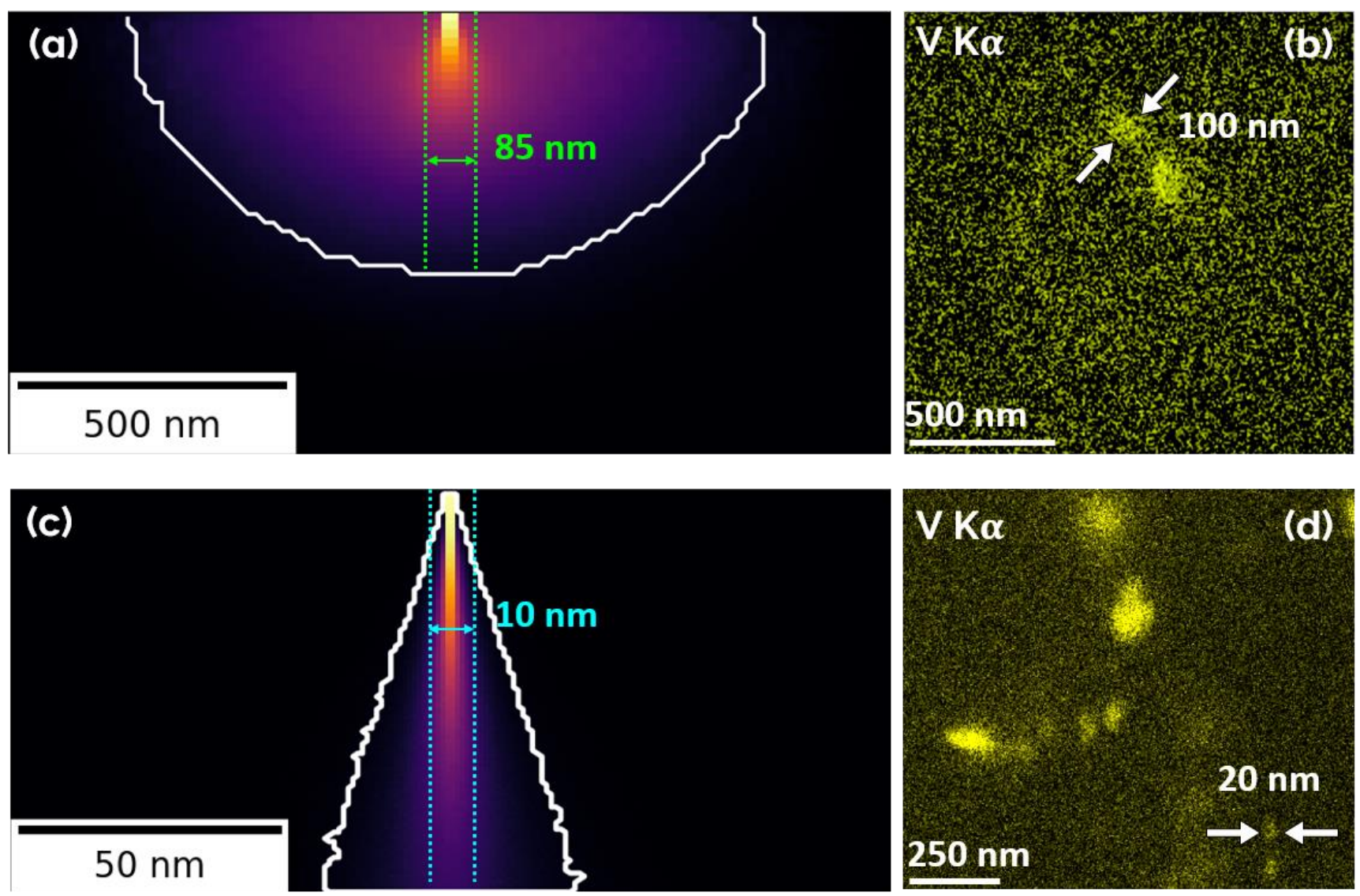

Figure 1. Monte Carlo simulations of V Ka X-ray generation for a bulk SEM sample analysed at 20kV, a), and a $100 \mathrm{~nm}$ thick STEM-SEM sample analysed at $30 \mathrm{kV}, \mathrm{c}$ ). The effective spatial resolution has been highlighted on each simulation. Experimental V Ka X-ray maps call attention to the differences in spatial resolution. Data was acquired from a steel sample at b) $20 \mathrm{kV}$ (SEM) and d) $30 \mathrm{kV}$ (STEM-SEM) with 30 minutes acquisition time.

\begin{tabular}{|c|cc|}
\hline $\begin{array}{c}\text { Accelerating } \\
\text { Voltage }\end{array}$ & Fe Ka & Fe La \\
\hline $\mathbf{2 ~ k V}$ & 0 & Counts $/($ s.nA.msr $)$ \\
\hline $\mathbf{3 0 ~ k V}$ & 3167.54 & 701.65 \\
\hline $\mathbf{2 0 0} \mathbf{~ k V}$ & 1352.73 & 917.54 \\
\hline
\end{tabular}

Figure 2. Theoretical X-ray detection of Fe K $\alpha$ and L $\alpha$ for at 2, 30 and $200 \mathrm{kV}$ accelerating voltage. 


\section{References}

[1] Burgess, S., Sagar, J., Holland, J., Li, X., \& Bauer, F. (2017). Ultra-low kV EDS-a new approach to improved spatial resolution, surface sensitivity, and light element compositional imaging and analysis in the SEM. Microscopy Today, 25(2), 20-29.

[2] Ritchie, N. W. (2005). A new Monte Carlo application for complex sample geometries. Surface and Interface Analysis: An International Journal devoted to the development and application of techniques for the analysis of surfaces, interfaces and thin films, 37(11), 1006-1011.

[3] Sagar, J., Wood, D., Pinard, P., Howe, J., Holland, J., Burgess, S., \& Statham, P. (2018). X-ray Spectrum Imaging at High Resolution in the STEM and STEM/SEM and SEM. Microscopy and Microanalysis, 24(S1), 656-657. 